\title{
ON MINIMAL SURFACES IN A KÄHLER MANIFOLD OF CONSTANT HOLOMORPHIC SECTIONAL CURVATURE
}

\author{
BY
}

JON G. WOLFSON

\begin{abstract}
This paper studies minimal surfaces in Kähler manifolds of constant holomorphic sectional curvature using the technique of the moving frame. In particular, we provide a classification of the minimal two-spheres in $\mathbf{C} P^{n}$, complex projective $n$-space, equipped with the Fubini-Study metric. This classification can be described as follows: To each holomorphic curve in $\mathbf{C} P^{n}$ classically there is associated a particular framing of $\mathbf{C}^{n+1}$ called the Frenet frame. Each element of the Frenet frame induces a minimal surface in $C P^{n}$. The classification theorem states that all minimal surfaces of topological type of the two-sphere occur in this manner. The theorem is proved using holomorphic differentials that occur naturally on minimal surfaces in Kähler manifolds of constant holomorphic sectional curvature together with the Riemann-Roch Theorem.
\end{abstract}

1. Introduction. In this paper we study, using moving frames, minimal surfaces in complex projective space (with the Fubini-Study metric) and, more generally, minimal surfaces in Kähler manifolds of constant holomorphic sectional curvature.

On a minimal surface in any Kähler manifold an invariant cubic form appears naturally [7]. If the ambient Kähler manifold is of constant holomorphic sectional curvature, then this form is holomorphic. Moreover, if this cubic form vanishes (as it will, for example, if the surface is a two-sphere), then one or possibly two holomorphic quartic forms appear on the surface. If these vanish, then one or possibly two quintic holomorphic forms appear and so on. The vanishing of all these forms allows us to construct a special framing along the surface known as a Frenet frame. This generalizes the classical construction of Frenet frames along holomorphic curves in projective space. In the case that the minimal surface lies in projective space the existence of a Frenet frame along the surface allows us to show that the surface can be constructed from a certain unique holomorphic curve via a process involving basically only differentiation. It will follow that all minimal two-spheres in projective space can be constructed, via this procedure, from holomorphic curves.

The classification of minimal two-spheres in complex projective space was first carried out by Din and Zakrzewski [8]. Somewhat later in [9] Eells and Wood gave a mathematically rigorous treatment of the work of Din and Zakrzewski with some interesting extensions to the case of minimal tori in $\mathbf{C P} P^{n}$. This work and [7] follow Eells and Wood though they are independent of them. In fact they grew out of an

Received by the editors April 23, 1984 and, in revised form, September 11, 1984.

1980 Mathematics Subject Classification. Primary 53A10; Secondary 53C55. 
attempt to understand the results of Din and Zakrzewski from the point of view of the moving frame.

In $\$ 2$ we derive the fundamental equations of a minimal surface in a Kähler manifold. In $\$ 3$ we apply these equations to construct a Frenet frame along a minimal two-sphere in a Kähler manifold of constant holomorphic sectional curvature. $\S 4$ discusses minimal surfaces in complex projective space. We conclude in $\$ 5$ with a few remarks.

I would like to express my gratitude to Professor S. S. Chern for his advice, encouragement and support during the completion of this work.

2. Minimal surfaces in a Kähler manifold. Consider a Kähler manifold $X$ of complex dimension $n$. We write the metric of $X$

$$
d s^{2}=\sum \omega_{\alpha} \bar{\omega}_{\alpha}=\sum \omega_{\alpha} \omega_{\bar{\alpha}} .
$$

Here and throughout this paper we employ the index conventions

$$
\alpha, \beta=-1,1,2, \ldots, n-1, \quad \lambda, \mu=2, \ldots, n-1 .
$$

The forms $\omega_{\alpha}$ are of type $(1,0)$ and are defined up to a unitary transformation. They constitute a unitary coframe. Relative to a coframe field $\omega_{\alpha}$ a unitary connection $\omega_{\alpha \bar{\beta}}$ is uniquely determined by the conditions

$$
\begin{gathered}
d \omega_{\alpha}=\sum \omega_{\alpha \bar{\beta}} \wedge \omega_{\beta}, \\
\omega_{\alpha \bar{\beta}}+\omega_{\bar{\beta} \alpha}=0 \quad\left(\omega_{\bar{\beta} \alpha}=\bar{\omega}_{\beta \bar{\alpha}}\right) .
\end{gathered}
$$

If $e_{-1}, \ldots, e_{n-1}$ is the unitary frame dual to $\omega_{-1}, \ldots, \omega_{n-1}$, then we can write the covariant derivative of $e_{\alpha}$ as

$$
D e_{\alpha}=\sum \bar{\omega}_{\alpha \bar{\beta}} e_{\beta},
$$

where $\omega_{\alpha \bar{\beta}}$ is the connection satisfying (2.3) and (2.4). The curvature of the metric (2.1) is given by

$$
d \omega_{\alpha \bar{\beta}}=\sum \omega_{\alpha \bar{\gamma}} \wedge \omega_{\gamma \bar{\beta}}+\Omega_{\alpha \bar{\beta}}
$$

$X$ is said to be of constant holomorphic sectional curvature $4 \rho$ if

$$
\Omega_{\alpha \bar{\beta}}=-\rho\left(\omega_{\alpha} \wedge \omega_{\bar{\beta}}+\delta_{\alpha \bar{\beta}} \sum \omega_{\gamma} \wedge \omega_{\bar{\gamma}}\right) .
$$

Consider now an immersed surface

$$
x: M \rightarrow X
$$

We can choose a field of coframes over $M$ satisfying

$$
\omega_{\lambda}=0 \text {. }
$$

The induced metric on $M$ is then

$$
d s_{x}^{2}=\omega_{-1} \bar{\omega}_{-1}+\omega_{1} \bar{\omega}_{1} .
$$

Let the complex valued 1-form $\phi$ define the complex structure on $M$. We can modify $\phi$ by a real factor so that

$$
d s_{x}^{2}=\phi \bar{\phi}
$$


$\phi$ is then defined up to a complex factor of norm 1. We have, restricted to $M$,

$$
\omega_{i}=s_{i} \phi+t_{i} \bar{\phi}, \quad i=1,-1,
$$

where $s_{i}$ and $t_{i}$ are complex valued smooth functions on $M$. Substituting (2.12) into (2.10) and comparing with (2.11) we get

$$
\left|s_{1}\right|^{2}+\left|s_{-1}\right|^{2}+\left|t_{1}\right|^{2}+\left|t_{-1}\right|^{2}=1, \quad s_{1} \bar{t}_{1}+s_{-1} \bar{t}_{-1}=0 \text {. }
$$

The $\omega_{i}$ are defined up to a unitary transformation. In $\mathbf{C}^{2}$ we introduce the vectors $\vec{s}=\left(s_{1}, s_{-1}\right)$ and $\vec{t}=\left(t_{1}, t_{-1}\right)$. Equations (2.13) show that they are orthogonal, with the sum of the squares of their norms equal to 1 . By a unitary transformation at each point of $M$ we can suppose $\vec{s}=(s, 0)$ and $\vec{t}=(0, t)$, giving the "normalization"

$$
\omega_{1}=s \phi, \quad \omega_{-1}=t \bar{\phi},
$$

where $s$ and $t$ are complex valued smooth functions which satisfy $|s|^{2}+|t|^{2}=1$.

The notion of a minimal surface in $X$ is defined in terms of its underlying Riemannian structure. We will find the condition that $x: M \rightarrow X$ is a minimal surface. We set

$$
\begin{aligned}
& \bar{s} \omega_{1}+t \bar{\omega}_{-1}=\theta_{1}+\sqrt{-1} \theta_{2}, \\
& \bar{t} \omega_{1}-s \bar{\omega}_{-1}=\theta_{3}+\sqrt{-1} \theta_{4}, \\
& \omega_{\lambda}=\theta_{2 \lambda+1}+\sqrt{-1} \theta_{2 \lambda+2} .
\end{aligned}
$$

Then $\theta_{A}, 1 \leqslant A \leqslant 2 n$, is an orthonormal coframe of the underlying Riemannian structure of $X$. It is also a Darboux coframe of $M$, because along $M$ we have

$$
\begin{gathered}
\bar{t} \omega_{1}-s \bar{\omega}_{-1}=0, \\
\omega_{\lambda}=0 .
\end{gathered}
$$

By taking the exterior derivative of (2.16) and making use of (2.3) and (2.14), we get

$$
\left[(s d \bar{t}-\bar{t} d s)+s \bar{t}\left(\omega_{1 \overline{1}}+\omega_{-1,-\overline{1}}\right)\right] \wedge \phi+\omega_{1,-\overline{1}} \wedge \bar{\phi}=0,
$$

which allows us to set

$$
\begin{gathered}
(s d \bar{t}-\bar{t} d s)+s \bar{t}\left(\omega_{1 \overline{1}}+\omega_{-1,-\overline{1}}\right)=a \phi+b \bar{\phi}, \\
\omega_{1,-\overline{1}}=b \phi+c \bar{\phi},
\end{gathered}
$$

where $a, b$ and $c$ are complex valued functions. Similarly, exterior differentiation of (2.17) gives

$$
s \omega_{\lambda \overline{1}} \wedge \phi+t \omega_{\lambda,-\overline{1}} \wedge \bar{\phi}=0
$$

and we can write

$$
s \omega_{\lambda \overline{1}}=a_{\lambda} \phi+b_{\lambda} \bar{\phi}, \quad t \omega_{\lambda,-\overline{1}}=b_{\lambda} \phi+c_{\lambda} \bar{\phi},
$$

where $a_{\lambda}, b_{\lambda}$ and $c_{\lambda}$ are complex valued functions.

Instead of the $2 n-2$ real second fundamental forms we can consider the $n-1$ complex valued ones:

$$
\mathrm{II}^{\mathbf{C}}=a \phi^{2}+2 b \phi \bar{\phi}+c \bar{\phi}^{2}, \quad \mathrm{II}_{\lambda}^{\mathrm{C}}=a_{\lambda} \phi^{2}+2 b_{\lambda} \phi \bar{\phi}+c_{\lambda} \bar{\phi}^{2}
$$


To see the relation between the real second fundamental forms and $\mathrm{II}^{\mathbf{C}}$ and $\mathrm{II}_{\lambda}^{\mathrm{C}}$ we must compare the Kähler connection $\omega_{\alpha \bar{\beta}}$ with the Levi-Civita connection $\theta_{A B}$ of the Darboux coframe (2.15). For example, the equation

$$
\omega_{\lambda}=\theta_{2 \lambda+1}+\sqrt{-1} \theta_{2 \lambda+2}
$$

gives on the one hand (2.20). On the other hand, along $M$ we have $\theta_{2 \lambda+1}=0$, and $\theta_{2 \lambda+2}=0$. Taking the exterior derivative we obtain

$$
\begin{aligned}
& 0=d \theta_{2 \lambda+1}=\theta_{2 \lambda+1,1^{\wedge}} \theta_{1}+\theta_{2 \lambda+1,2^{\wedge}} \theta_{2}, \\
& 0=d \theta_{2 \lambda+2}=\theta_{2 \lambda+2,1^{\wedge}} \theta_{1}+\theta_{2 \lambda+2,2^{\wedge}} \theta_{2} .
\end{aligned}
$$

By Cartan's Lemma

$$
\begin{aligned}
& \theta_{2 \lambda+i, 1}=\alpha_{2 \lambda+i} \theta_{1}+\beta_{2 \lambda+i} \theta_{2}, \\
& \theta_{2 \lambda+i, 2}=\beta_{2 \lambda+i} \theta_{1}+\gamma_{2 \lambda+i} \theta_{2},
\end{aligned} \quad i=1,2 .
$$

Thus we get the second fundamental forms

$$
\mathrm{II}_{2 \lambda+i}=\alpha_{2 \lambda+i} \theta_{1}^{2}+2 \beta_{2 \lambda+i} \theta_{1} \theta_{2}+\gamma_{2 \lambda+i} \theta_{2}^{2}, \quad i=1,2 .
$$

By comparing both sides of the exterior derivative of $(2.15)(\mathrm{c})$, it follows easily that

$$
\begin{gathered}
\left(\begin{array}{cc}
1 & 1 \\
\sqrt{-1} & -\sqrt{-1}
\end{array}\right)\left(\begin{array}{ll}
a_{\lambda} & b_{\lambda} \\
b_{\lambda} & c_{\lambda}
\end{array}\right)\left(\begin{array}{cc}
1 & \sqrt{-1} \\
1 & -\sqrt{-1}
\end{array}\right) \\
=\left(\begin{array}{ll}
\alpha_{2 \lambda+1} & \beta_{2 \lambda+1} \\
\beta_{2 \lambda+1} & \gamma_{2 \lambda+1}
\end{array}\right)+\sqrt{-1}\left(\begin{array}{cc}
\alpha_{2 \lambda+2} & \beta_{2 \lambda+2} \\
\beta_{2 \lambda+2} & \gamma_{2 \lambda+2}
\end{array}\right) .
\end{gathered}
$$

The analogous result for II $^{\mathrm{C}}$ follows by considering the second equation of (2.15).

The condition for $M$ to be minimal is the vanishing of the traces of $\operatorname{II}^{\mathbf{C}}, \mathrm{II}_{\lambda}^{\mathbf{C}}$, which is

$$
b=b_{\lambda}=0 .
$$

In the case that $M$ is minimal (2.18) yields interesting information about the zeros of $s$ and $t$. Let $p \in M$; then as $|s|^{2}+|t|^{2}=1$, in a neighborhood of $p$ either $s \neq 0$ or $t \neq 0$, say $s \neq 0$. (2.18) can then be written

$$
d(s \bar{t})+s \bar{t}\left(\omega_{1 \overline{1}}+\omega_{-1,-\overline{1}}-2 \frac{d s}{s}\right)=a \phi
$$

Suppose $\zeta$ is a complex coordinate centered at $p$. Then (2.22) implies

$$
\frac{\partial(s \bar{t})}{\partial \bar{\zeta}}+s \bar{t} \cdot h=0,
$$

where $h$ is a $C^{\infty}$ complex valued function. In fact $h d \bar{\zeta}$ is the $(0,1)$ part of $\omega_{1 \overline{1}}+\omega_{-1,-\overline{1}}-2 d s / 2$. By a result in [5] (see our Theorem 3.2) (2.23) implies that either $s \bar{t}$ vanishes identically or $s \bar{t}=\zeta^{r} k(\zeta)$, where $r$ is an integer $\geqslant 0$ and $k$ is a $C^{\infty}$ complex valued function such that $k(0) \neq 0$. In particular if $M$ is minimal, then $s$ and $t$ either vanish identically (in which case $M$ is a holomorphic or antiholomorphic curve) or they have only isolated zeros.

In our investigations in $\S 4$, minimal surfaces will arise for which $s$ and $t$ both vanish at isolated points of the surface. At all points, however, $s$ and $t$ will satisfy

$$
s=\zeta^{q} g(\zeta), \quad t=\zeta^{r} h(\zeta)
$$


where $q$ and $r$ are integers $\geqslant 0$ and $g$ and $h$ are $C^{\infty}$ complex valued functions such that $g(0) \neq 0$ and $h(0) \neq 0$. Points where both $s$ and $t$ vanish (i.e., points $\zeta=0$ where $q$ and $r$ are $\geqslant 1$ in (2.24)) are called branch points of the minimal surface and such minimal surfaces are called branched minimal surfaces. It will prove useful to include branched minimal surfaces in our study, so for the remainder of this paper by minimal surface we will mean branched minimal surface, unless stated otherwise.

When $M$ is minimal and $X$ has constant holomorphic sectional curvature, (2.19) leads to an interesting theorem. Our coframe is defined up to the transformation

$$
\omega_{1} \mapsto e^{i k_{1}} \omega_{1}, \quad \omega_{-1} \mapsto e^{i k_{-1}} \omega_{-1}, \quad k_{1}, k_{-1} \text { real, }
$$

under which $\omega_{1,-\overline{1}}$ transforms as

$$
\omega_{1,-\overline{1}} \mapsto e^{i k_{1}} \omega_{1,-\overline{1}} e^{-i k_{-1}}
$$

Hence the complex valued symmetric differential form of type $(3,0)$

$$
\Lambda_{0}=\omega_{1} \omega_{\overline{1},-1} \omega_{-\overline{1}}=s \bar{t} \bar{c} \phi^{3},
$$

is an invariant of the minimal surface $M$.

THEOREM 2.1. Let $X$ be a Kähler manifold of constant holomorphic sectional curvature. Let $M \rightarrow X$ be a minimal surface. Then the cubic form $\Lambda_{0}$ is holomorphic.

Proof. The conclusion means that relative to a local coordinate $\zeta$ of the complex structure $\phi$ on $M, \Lambda_{0}=f(\zeta) d \zeta^{3}$, where $f(\zeta)$ is a holomorphic function of $\zeta$.

Let

$$
\omega_{1}=p_{1} d \zeta, \quad \omega_{-1}=p_{-1} d \bar{\zeta}, \quad \omega_{1,-\overline{1}}=q d \bar{\zeta},
$$

so that $\Lambda_{0}=\left(p_{1} \bar{p}_{-1} \bar{q}\right) \zeta^{3}$. We must show that the product in the parentheses is a holomorphic function of $\zeta$.

The structure equations give

$$
d \omega_{1,-\overline{1}}=\left(\omega_{1, \overline{1}}-\omega_{-1, \overline{1}}\right) \wedge \omega_{1,-\overline{1}}+\sum \omega_{1 \bar{\lambda}} \wedge \omega_{\lambda,-\overline{1}}+\Omega_{1,-\overline{1}}
$$

By (2.20) $\omega_{1 \bar{\lambda}}=-\bar{\omega}_{\lambda \overline{1}}$ and $\omega_{\lambda,-\overline{1}}$ are, for a minimal surface, both of type $(0,1)$ so that the middle sum is zero. On the other hand, $\Omega_{1,-\overline{1}}=0$ since $X$ is of constant holomorphic sectional curvature. Substituting the expression for $\omega_{1,-\overline{1}}$ into this equation, we get

$$
d \bar{q} \equiv \bar{q}\left(\omega_{-1,-\overline{1}}-\omega_{1, \overline{1}}\right) \quad \bmod d \zeta .
$$

Similarly, by using the formulas for $d \omega_{1}, d \omega_{-1}$, we derive

$$
d p_{1} \equiv p_{1} \omega_{1, \overline{1}}, \quad d \bar{p}_{2} \equiv-\bar{p}_{2} \omega_{-1,-\overline{1}} \quad \bmod d \zeta .
$$

Hence we get $d\left(p_{1} \bar{p}_{-1} \bar{q}\right) \equiv 0 \bmod d \zeta$, and the theorem is proved.

COROLlaRY 2.1. Under the assumptions of the theorem, if $M$ is the two-sphere $S^{2}$, then $\Lambda_{0}=0$.

Proof. The Riemann-Roch Theorem implies that there are no nonzero holomorphic forms on $S^{2}$. 
3. The Frenet frame of a minimal two-sphere in a Kähler manifold of constant holomorphic sectional curvature. Let $X$ be a Kähler manifold of dimension $n, x$ : $M \rightarrow X$ an immersed surface and $U \subseteq M$ a neighborhood in $M$.

DEFINITION 3.1. A unitary frame for $U \subseteq M$ along $x$ is a set of $n C^{\infty}$ mappings

$$
e_{\alpha}: U \subseteq M \rightarrow T_{*} X, \quad \alpha=-1,1, \ldots, n-1,
$$

such that $e_{-1}(p), e_{1}(p), \ldots, e_{n-1}(p)$ is a unitary basis for $T_{x(p)} X$ for each $p \in U$.

For brevity we often suppress mention of $U$ or $M$ and say "a unitary frame along $x$ ".

Suppose now that $x: M \rightarrow X$ is a branched minimal immersion which is neither holomorphic nor antiholomorphic. We saw in $\$ 2$ that there is a unitary coframe $\omega_{-1}, \omega_{1}, \ldots, \omega_{n-1}$ on $M$ such that

$$
\omega_{1}=s \phi, \quad \omega_{-1}=t \bar{\phi}, \quad \omega_{\lambda}=0,
$$

where $s$ and $t$ satisfy (2.24). Let $e_{1}$ (respectively $e_{-1}$ ) be the vector field dual to $\omega_{1}$ (respectively $\omega_{-1}$ ). Then $e_{1}$ (resp. $e_{-1}$ ) is a well-defined $C^{\infty}$ mapping $U \subseteq M \rightarrow T_{*} X$, except at the isolated points where $s=0$ (resp. $t=0$ ). However, because $s$ and $t$ both satisfy (2.24) the maps $e_{1}$ and $e_{-1}$ can be smoothly extended across their singularities to form smooth vector fields. $e_{-1}, e_{1}$ can now be extended to a unitary frame $e_{-1}, e_{1}, e_{2}, \ldots, e_{n-1}$ along $x$. We shall only consider unitary frames along $x$ for which $e_{-1}$ and $e_{1}$ have been chosen in this manner.

The covariant differential of the field $e_{\alpha}$ is given by

$$
D e_{\alpha}=\sum_{\beta} x^{*}\left(\bar{\omega}_{\alpha \bar{\beta}}\right) e_{\beta},
$$

where the $\omega_{\alpha \bar{\beta}}$ are the connection forms of the unitary coframe dual to $\left\{e_{-1}, e_{1}, \ldots, e_{n-1}\right\}$. We will denote (3.1) by

$$
D e_{\alpha}=\sum_{\beta} \bar{\omega}_{\alpha \bar{\beta}} e_{\beta} .
$$

Definition 3.2. We say that the fields $\left\{e_{1}, \ldots, e_{k}\right\}$ for some $k, 1 \leqslant k \leqslant n-1$, form a Frenet framing of the holomorphic osculating space of $x$ if they satisfy the properties:

(1) $\left\{e_{-1}, e_{1}, e_{2}, \ldots, e_{k}, \ldots, e_{n-1}\right\}$ is a unitary frame along $x$,

(2) $D e_{1}=\bar{\omega}_{1 \overline{1}} e_{1}+\bar{\omega}_{1 \overline{2}} e_{2}$,

(3) $D e_{\gamma}=\bar{\omega}_{\gamma, \overline{\gamma-1}} e_{\gamma-1}+\bar{\omega}_{\gamma, \bar{\gamma}} e_{\gamma}+\bar{\omega}_{\gamma, \overline{\gamma+1}} e_{\gamma+1}$ for $1<\gamma<k$,

(4) $D e_{k}=\bar{\omega}_{k, \overline{k-1}} e_{k-1}+\bar{\omega}_{k, \bar{k}} e_{k}$,

where the forms $\bar{\omega}_{1 \overline{2}}$ and $\bar{\omega}_{\gamma, \bar{\gamma}+1}, 1<\gamma<k$, are of type $(1,0)$ and not identically zero.

There is a similar definition for a Frenet framing of the antiholomorphic osculating space of $x$.

If $y: M \rightarrow X$ is a holomorphic curve in the Kähler manifold $X$, then the "normalization" (2.14) becomes

$$
\omega_{1}=s \phi, \quad \omega_{-1}=0, \quad \omega_{\lambda}=0
$$


and so the vector field $e_{-1}$ is undefined. Making this modification to Definition 3.2, in [6] it is shown that the holomorphic osculating space of the holomorphic curve $y$ (and so the total osculating space of $y$ ) admits a Frenet framing if the Kähler manifold $X$ is of constant holomorphic sectional curvature. We will prove the following partial generalization.

THEOREM 3.1. Let $x: S^{2} \rightarrow X$ be a branched minimal immersion which is neither holomorphic nor antiholomorphic, where $X$ is a Kähler manifold of constant holomorphic sectional curvature. Then there exists a Frenet framing of the holomorphic osculating space of $x$.

To prove this theorem we will need the following result [5]:

THEOREM 3.2. Let $h_{\eta}(\zeta, \bar{\zeta})$ be complex valued functions which satisfy the differential system

$$
\frac{\partial h_{\eta}}{\partial \zeta}=\sum_{\theta} a_{\eta \theta} h_{\theta}, \quad 1 \leqslant \eta, \theta \leqslant m,
$$

in a neighborhood of $\zeta=0$, where the $a_{\eta \theta}$ are complex valued $C^{1}$ functions. Suppose that the $h_{\eta}$ do not all vanish identically. Then the $h_{\eta}$ are of the form

$$
h_{\eta}(\zeta)=\bar{\zeta} \tilde{h}_{\eta}(\zeta)
$$

where $r$ is an integer $\geqslant 0$ and the $\tilde{h}_{\eta}(0)$ are not all zero.

Proof of Theorem 3.1. Let $\left\{e_{-1}, e_{1}, \ldots, e_{n-1}\right\}$ be a unitary frame along $x$, as constructed above, and let $\left\{\omega_{-1}, \omega_{1}, \ldots, \omega_{n-1}\right\}$ be the dual coframe. We have

$$
D e_{1}=\bar{\omega}_{1, \overline{1}} e_{-1}+\bar{\omega}_{1 \overline{1}} e_{1}+\sum_{\lambda} \bar{\omega}_{1 \bar{\lambda}} e_{\lambda}
$$

where the $\omega_{\alpha \bar{\beta}}$ are the connection forms of the coframe $\left\{\omega_{-1}, \ldots, \omega_{n-1}\right\}$. The holomorphic $(3,0) \Lambda_{0}$ defined in $\S 2$ is $\Lambda_{0}=\omega_{1} \omega_{\overline{1},-1} \omega_{-\overline{1}}$. By Corollary $2.1, \Lambda_{0}=0$. It follows that

$$
\omega_{1,-\overline{1}}=0 \text {. }
$$

On the other hand, from (2.20) it follows that $\omega_{\lambda \overline{1}}$ is a $(1,0)$ form. Let $\zeta$ be a local complex coordinate. We can write

$$
\omega_{1 \bar{\lambda}}=h_{\bar{\lambda}} d \bar{\zeta}
$$

where the $h_{\bar{\lambda}}$ are $C^{\infty}$ complex valued functions. (3.4) becomes

$$
D e_{1}=\bar{\omega}_{1 \overline{1}} e_{1}+d \zeta\left(\sum \bar{h}_{\bar{\lambda}} e_{\lambda}\right) \text {. }
$$

Taking the exterior derivative of (3.6) and using the structure equations we get

$$
\begin{aligned}
d h_{\bar{\lambda}} d \bar{\zeta} & =\omega_{1,-\overline{1}} \wedge \omega_{-1, \bar{\lambda}}+\omega_{1 \overline{1}} \wedge \omega_{1 \bar{\lambda}}+\sum \omega_{1 \bar{\mu}} \wedge \omega_{\mu \bar{\lambda}}+\Omega_{1 \bar{\lambda}} \\
& =\left(h_{\bar{\lambda}} \omega_{1 \overline{1}}-\sum h_{\bar{\mu}} \omega_{\mu \bar{\lambda}}\right) \wedge d \bar{\zeta} .
\end{aligned}
$$

This means that the $h_{\bar{\lambda}}$ satisfy a differential system of the type $\partial h_{\bar{\lambda}} / \partial \zeta=\sum a_{\bar{\lambda} \mu} h_{\bar{\mu}}$, where the $a_{\bar{\lambda} \mu}$ are $C^{\infty}$ complex valued functions. From Theorem 3.2 we can conclude either that all the $h_{\bar{\lambda}}$ vanish identically or that the $h_{\bar{\lambda}}$ are of the form (3.3). 
In the former case, $D e_{1}=\bar{\omega}_{1 \overline{1}} e_{1}$ and the proof of the theorem is complete. In the latter case we can make a unitary change of frame so that $\omega_{12}$ is a $(0,1)$ form with only isolated zeros and $\omega_{1 \bar{v}}=0$, for $3 \leqslant v \leqslant n-1$. In this case (3.7) becomes

$$
D e_{1}=\bar{\omega}_{1 \overline{1}} e_{1}+\bar{\omega}_{1 \overline{2}} e_{2} \text {. }
$$

Now by way of induction we suppose that for some $j, 1 \leqslant j<n-2$, we have constructed $C^{\infty}$ vector fields $e_{2}, \ldots, e_{j+1}$ along $x$ such that:

(1) $\left\{e_{-1}, e_{1}, e_{2}, \ldots, e_{j+1}\right\}$ is part of a unitary frame along $x$,

(2) $D e_{1}=\bar{\omega}_{1 \overline{1}} e_{1}+\bar{\omega}_{12} e_{2}$,

$$
\begin{aligned}
& D e_{2}=\bar{\omega}_{2 \overline{1}} e_{1}+\bar{\omega}_{2 \overline{2}} e_{2}+\bar{\omega}_{2 \overline{3}} e_{3}, \\
& \vdots \\
& D e_{j-1}=\bar{\omega}_{j-1}, \overline{j-2} e_{j-2}+\bar{\omega}_{j-1, \overline{j-1}} e_{j-1}+\bar{\omega}_{j-1, j} e_{j}, \\
& D e_{j}=\bar{\omega}_{j, \overline{j-1}} e_{j-1}+\bar{\omega}_{j, j} e_{j}+\bar{\omega}_{j, \overline{j+1}} e_{j+1},
\end{aligned}
$$

where $\bar{\omega}_{\gamma, \overline{\gamma+1}}$ is a $(1,0)$ form with only isolated zeros for $1 \leqslant \gamma \leqslant j$.

We now complete $\left\{e_{-1}, e_{1}, \ldots, e_{j+1}\right\}$ to a unitary frame $\left\{e_{-1}, e_{1}, \ldots, e_{n-1}\right\}$ along $x$ and let $\left\{\omega_{-1}, \omega_{1}, \ldots, \omega_{n-1}\right\}$ be the dual coframe. Let $\omega_{\alpha \bar{\beta}}$ be the connection forms of the coframe $\left\{\omega_{-1}, \omega_{1}, \ldots, \omega_{n-1}\right\}$. Consider

$$
D e_{j+1}=\bar{\omega}_{j+1,-\overline{1}} e_{-1}+\bar{\omega}_{j+1, \overline{1}} e_{1}+\sum_{\lambda} \bar{\omega}_{j+1, \bar{\lambda}} e_{\lambda} .
$$

It follows from (3.9) that $\omega_{j+1, \bar{\gamma}}=0$ for $1 \leqslant \gamma \leqslant j-1$, so

$$
D e_{j+1}=\bar{\omega}_{j+1,-\overline{1}} e_{-1}+\bar{\omega}_{j+1, j} e_{j}+\bar{\omega}_{j+1, \overline{j+1}} e_{j+1}+\sum_{\nu} \bar{\omega}_{j+1, \bar{\nu}} e_{\nu},
$$

where here and for the remainder of this section we use the index range $j+2 \leqslant \nu$, $\xi \leqslant n-1$.

In order to accomplish the inductive step it will be necessary to show that $\omega_{j+1,-\overline{1}}=0$. As was the case in showing that $\omega_{1,-i}=0$ this requires the introduction of a global invariant on $S^{2}$. To this end we note, by (3.9), $\omega_{\bar{\gamma}, \gamma+1}, 1 \leqslant \gamma \leqslant j$, are $(1,0)$ forms and, by $(2.20), \omega_{j+1},-1$ is also a $(1,0)$ form. This allows us to define a complex valued symmetric form of bidegree $(j+3,0)$

DeFinition 3.3. $\Lambda_{j}=\omega_{1} \omega_{\overline{1} 2} \omega_{\overline{2} 3} \cdots \omega_{j, j+1} \omega_{\overline{j+1},-1} \omega_{-\overline{1}}$.

$\Lambda_{j}$ is a generalization of the symmetric $(3,0)$ form $\Lambda_{0}$. Of course, $\Lambda_{j}$ is only defined locally. A priori, it depends on the choice of unitary frame $\left\{e_{-1}, e_{1}, \ldots, e_{n-1}\right\}$. However, we have

Proposition 3.1. $\Lambda_{j}$ is invariantly defined and so $\Lambda_{j}$ is a globally defined symmetric form on $S^{2}$.

Furthermore, we have the crucial

THEOREM 3.3. $\Lambda_{j}$ is a holomorphic form.

We will postpone the proof of these results in order to complete the induction.

Corollary 3.1. $\Lambda_{j}=0$. 
Proof. The Riemann-Roch Theorem applies as in the proof of Corollary 2.1.

By the inductive assumption, the forms $\omega_{\bar{\gamma}, \gamma+1}$ have only isolated zeros. It follows then from the corollary that

$$
\omega_{j+1,-\overline{1}}=0
$$

Hence (3.10) becomes

$$
D e_{j+1}=\bar{\omega}_{j+1, j} e_{j}+\bar{\omega}_{j+1, \overline{j+1}}+\sum_{\nu} \bar{\omega}_{j+1, \bar{\nu}} e_{\nu} .
$$

We must now deal with the terms $\omega_{j+1, \bar{\nu}}$ in (3.12). From (3.9) we have

$$
\omega_{j \bar{\nu}}=0 \text {. }
$$

Taking the exterior derivative of (3.13) and using the structure equations, the curvature assumption and (3.9), we get

$$
0=d \omega_{j \bar{\nu}}=\omega_{j, \overline{j+1}} \wedge \omega_{j+1, \nu} .
$$

By the inductive hypothesis $\omega_{j, \overline{j+1}}$ is a $(0,1)$ form, so the $\omega_{j+1, \bar{\nu}}$ are also $(0,1)$ forms. We can write

$$
\omega_{j+1, \bar{\nu}}=g_{\bar{\nu}} d \bar{\zeta}
$$

where the $g_{\bar{\nu}}$ are $C^{\infty}$ complex valued functions. Taking the exterior derivative of (3.14) and using the curvature assumption, (3.9) and (3.11) we have

$$
\begin{aligned}
d g_{\bar{\nu}} \wedge d \bar{\zeta} & =d \omega_{j+1, \bar{\nu}}=\omega_{j+1, \overline{j+1}} \wedge \omega_{j+1, \bar{\nu}}+\sum_{\xi} \omega_{j+1, \bar{\xi}} \wedge \omega_{\xi, \bar{\nu}} \\
& =\left(g_{\bar{\nu}} \omega_{j+1, \overline{j+1}}-\sum_{\xi} g_{\bar{\xi}} \omega_{\xi, \bar{\nu}}\right) \wedge d \bar{\zeta} .
\end{aligned}
$$

This means that the $g_{\bar{\nu}}$ satisfy a differential system of the type

$$
\frac{\partial g_{\bar{\nu}}}{\partial \bar{\zeta}}=\sum b_{\bar{\nu} \xi} g_{\bar{\xi}}
$$

where the $b_{\bar{\nu} \xi}$ are $C^{\infty}$ complex valued functions. It follows from Theorem 3.2 that either the $g_{\bar{\nu}} \equiv 0$ or $g_{\bar{\nu}}(\zeta)=\bar{\zeta}^{s} \tilde{g}_{\bar{\nu}}(\zeta)$, where $s$ is an integer $\geqslant 0$ and the $\tilde{g}_{\bar{\nu}}(0)$ are not all zero. In the former case we have

$$
D e_{j+1}=\bar{\omega}_{j+1, j} e_{j}+\bar{\omega}_{j+1, \overline{j+1}} e_{j+1} .
$$

In the latter case we can make a unitary change of frame so that $\omega_{j+1, \overline{j+2}}$ is a $(0,1)$ form with only isolated zeros and so that $\omega_{j+1, \bar{\pi}}=0$ for $j+3 \leqslant \pi \leqslant n-1$. In this case we have

$$
D e_{j+1}=\bar{\omega}_{j+1, j} e_{j}+\bar{\omega}_{j+1, \overline{j+1}} e_{j+1}+\bar{\omega}_{j+1, \overline{j+2}} e_{j+2} .
$$

This completes the induction and the proof modulo Proposition 3.1 and Theorem 3.3.

Proof of Proposition 3.1. $\Lambda_{j}$ is defined with respect to a unitary frame $\left\{e_{-1}, e_{1}, \ldots, e_{j+1}, \ldots, e_{n-1}\right\} . e_{-1}$ and $e_{1}$ are defined according to the convention discussed at the beginning of this section. It follows that they are determined up to 
the transformation

$$
e_{-1} \mapsto \tilde{e}_{-1}=\exp \left(\sqrt{-1} \kappa_{-1}\right) e_{-1}, \quad e_{1} \mapsto \tilde{e}_{1}=\exp \left(\sqrt{-1} \kappa_{1}\right) e_{1},
$$

where $\kappa_{-1}$ and $\kappa_{1}$ are real valued functions. The vector fields $e_{2}, \ldots, e_{j+1}$ are required to satisfy (3.9). This implies that they are determined up to the transformation

$$
e_{\gamma} \mapsto \tilde{e}_{\gamma}=\exp \left(\sqrt{-1} \kappa_{\gamma}\right) e_{\gamma}, \quad \gamma=2, \ldots, j+1,
$$

where the $\kappa_{\gamma}$ are $C^{\infty}$ real valued functions. Let $\left\{\tilde{\omega}_{-1}, \tilde{\omega}_{1}, \tilde{\omega}_{2}, \ldots, \tilde{\omega}_{j+1}\right\}$ be the 1 -forms dual to the vector fields $\left\{\tilde{e}_{-1}, \tilde{e}_{1}, \tilde{e}_{2}, \ldots, \tilde{e}_{j+1}\right\}$ and let $\tilde{\omega}_{\alpha \bar{\beta}}$ be the connection forms of the coframe $\left\{\tilde{\omega}_{-1}, \tilde{\omega}_{1}, \ldots, \tilde{\omega}_{j+1}, \omega_{j+2}, \ldots, \omega_{n-1}\right\}$. Then we have

$$
\begin{aligned}
\tilde{\omega}_{-1} & =\exp \left(-\sqrt{-1} \kappa_{-1}\right) \omega_{-1}, \quad \tilde{\omega}_{1}=\exp \left(-\sqrt{-1} \kappa_{1}\right) \omega_{1}, \\
\tilde{\omega}_{\gamma} & =\exp \left(-\sqrt{-1} \kappa_{\gamma}\right) \omega_{\gamma}, \quad \gamma=2, \ldots, j+1,
\end{aligned}
$$

from which it follows that

$$
\begin{aligned}
\tilde{\omega}_{\bar{\delta}, \delta+1} & =\exp \left(\sqrt{-1} \kappa_{\delta}\right) \omega_{\bar{\delta}, \delta+1} \exp \left(-\sqrt{-1} \kappa_{\delta+1}\right), \quad \delta=1, \ldots, j, \\
\tilde{\omega}_{\overline{j+1},-1} & =\exp \left(\sqrt{-1} \kappa_{j+1}\right) \omega_{\overline{j+1},-1} \exp \left(-\sqrt{-1} \kappa_{-1}\right) .
\end{aligned}
$$

Therefore if $\tilde{\Lambda}_{j}$ denotes the symmetric $(j+3,0)$ form defined using the frame $\left\{\tilde{e}_{-1}, \tilde{e}_{1}, \ldots, \tilde{e}_{j+1}, e_{j+1}, \ldots, e_{n-1}\right\}$ we have $\tilde{\Lambda}_{j}=\Lambda_{j}$ from (3.18) and (3.19). We remark that $\Lambda_{j}$ does not depend on the choice of vector fields $e_{j+2}, \ldots, e_{n-1}$. The proposition follows.

Proof of Theorem 3.3. Let $\zeta$ be a local complex coordinate. We can write

$$
\begin{gathered}
\omega_{1}=r d \zeta, \quad \omega_{\gamma, \overline{\gamma+1}}=p_{\gamma} d \bar{\zeta}, \quad 1 \leqslant \gamma \leqslant j, \\
\omega_{j+1,-\overline{1}}=q d \bar{\zeta}, \quad \omega_{-1}=s d \bar{\zeta},
\end{gathered}
$$

where $r, p_{\gamma}, q$ and $s$ are $C^{\infty}$ complex valued functions. We have

$$
\Lambda_{j}=\left(r \bar{p}_{1} \bar{p}_{2} \cdots \bar{p}_{j} \bar{q} \bar{s}\right) d \zeta^{j+3} .
$$

We must show that the product in the parentheses is a holomorphic function of $\zeta$.

The structure equations give

$$
d \omega_{\gamma, \overline{\gamma+1}}=\sum_{\alpha} \omega_{\gamma, \bar{\alpha}} \wedge \omega_{\alpha, \overline{\gamma+1}}+\Omega_{\gamma, \overline{\gamma+1}}, \quad 1 \leqslant \gamma \leqslant j .
$$

Using (3.9), (3.20) and the curvature assumption this becomes

$$
\begin{aligned}
d p_{\gamma} \wedge d \bar{\zeta} & =\omega_{\gamma, \bar{\gamma}} \wedge \omega_{\gamma, \overline{\gamma+1}}+\omega_{\gamma, \overline{\gamma+1}} \wedge \omega_{\gamma+1, \overline{\gamma+1}} \\
& =p_{\gamma}\left(\omega_{\gamma, \bar{\gamma}}-\omega_{\gamma+1, \overline{\gamma+1}}\right) \wedge d \bar{\zeta} .
\end{aligned}
$$

So

$$
d \bar{p}_{\gamma}=\bar{p}_{\gamma}\left(\omega_{\gamma+1, \overline{\gamma+1}}-\omega_{\gamma, \bar{\gamma}}\right) \quad \bmod d \zeta, \quad 1 \leqslant \gamma \leqslant j .
$$

Also from the structure equations we have

$$
d \omega_{j+1,-\overline{1}}=\sum_{\alpha} \omega_{j+1, \bar{\alpha}} \wedge \omega_{\alpha,-\overline{1}}+\Omega_{j+1,-\overline{1}} .
$$


Using (3.9), (3.20) and the curvature assumption this becomes

$$
\begin{aligned}
d \omega_{j+1,-\overline{1}}= & \omega_{j+1,-\overline{1}} \wedge \omega_{-1,-\overline{1}}+\omega_{j+1, \overline{j+1}} \wedge \omega_{j+1,-\overline{1}} \\
& +\sum_{\nu=j+1}^{n-1} \omega_{j+1, \bar{\nu}} \wedge \omega_{\nu,-\overline{1}} .
\end{aligned}
$$

From (3.14) we know that $\omega_{j+1, \bar{\nu}}$ is a $(0,1)$ form and from $(2.20)$ we know that $\omega_{\nu,-\overline{1}}$ is also a $(0,1)$ form. It follows that the sum in (3.21) vanishes. Using (3.20) we can conclude that

$$
d \bar{q} \equiv \bar{q}\left(\omega_{-1,-\overline{1}}-\omega_{j+1, \overline{j+1}}\right) \quad \bmod d \zeta .
$$

Similarly using the formulas for $d \omega_{1}$ and $d \omega_{-1}$ we derive

$$
d r \equiv r \omega_{1, \overline{1}} \quad \bmod d \zeta, \quad d \bar{s} \equiv-\bar{s} \omega_{-1,-\overline{1}} \quad \bmod d \zeta .
$$

From these it follows easily that

$$
d\left(r \bar{p}_{1} \bar{p}_{2} \cdots \bar{p}_{j+1} \bar{q} \bar{s}\right) \equiv 0 \quad \bmod d \zeta .
$$

This completes the proof.

Using the same techniques as we employed in the proof of Theorem 3.1 we can prove

THEOREM 3.4. Let $x: S^{2} \rightarrow X$ be a branched minimal immersion which is neither holomorphic nor antiholomorphic, where $X$ is a Kähler manifold of constant holomorphic section curvature. Then there exists a Frenet framing of the antiholomorphic osculating space of $x$.

The proof of Theorem 3.4 involves defining symmetric $(i+3,0)$ forms $\Lambda_{-i}$, $i=1,2, \ldots$.

Let $\langle$,$\rangle denote the Hermitian scalar product in T_{*}(X)$.

Proposition 3.2. If $\left\{e_{1}, \ldots, e_{k}\right\}$ and $\left\{e_{-1}, \ldots, e_{-l}\right\}$ are Frenet frames for the holomorphic and antiholomorphic osculating spaces, respectively, of a minimal surface $x: S^{2} \rightarrow X$, then $\left\langle e_{-\delta}, e_{\gamma}\right\rangle=0$ for all $1 \leqslant \gamma \leqslant k, 1 \leqslant \delta \leqslant l$.

Proof. If $l=1$ we are done. Suppose $l>1$ and consider $\left\langle e_{-1}, e_{\gamma}\right\rangle=0$ for $1 \leqslant \gamma \leqslant k$. Taking the exterior derivative we have

$$
\begin{aligned}
0= & d\left\langle e_{-1}, e_{\gamma}\right\rangle=\left\langle D e_{-1}, e_{\gamma}\right\rangle+\left\langle e_{-1}, D e_{\gamma}\right\rangle \\
= & \left\langle\bar{\omega}_{-1,-\overline{1}} e_{-1}+\bar{\omega}_{-1,-\overline{2}} e_{-2}, e_{\gamma}\right\rangle \\
& +\left\langle e_{-1}, \bar{\omega}_{\gamma+1, \overline{\gamma-1}} e_{\gamma-1}+\bar{\omega}_{\gamma, \bar{\gamma}} e_{\gamma}+\bar{\omega}_{\gamma+1, \overline{\gamma+1}} e_{\gamma+1}\right\rangle \\
= & \bar{\omega}_{-1,-\overline{2}}\left\langle e_{-2}, e_{\gamma}\right\rangle .
\end{aligned}
$$

As $\omega_{-1,-\overline{2}}$ vanishes only at isolated points, we must have $\left\langle e_{-2}, e_{\gamma}\right\rangle=0,1 \leqslant \gamma \leqslant k$.

By induction suppose $\left\langle e_{-\delta}, e_{\gamma}\right\rangle=0$ for $1 \leqslant \gamma \leqslant k, 1 \leqslant \delta \leqslant i$, where $i<l$. Then by the inductive assumption

$$
\begin{aligned}
0= & d\left\langle e_{-i}, e_{\gamma}\right\rangle \\
= & \left\langle\bar{\omega}_{-i,-\overline{(i-1)}} e_{-(i-1)}+\bar{\omega}_{-i,-i} e_{-i}+\omega_{-i,-(\overline{i+1})} e_{-(i+1)}, e_{\gamma}\right\rangle \\
& +\left\langle e_{-i}, \bar{\omega}_{\gamma+1, \overline{\gamma-1}} e_{\gamma-1}+\bar{\omega}_{\gamma, \bar{\gamma}} e_{\gamma}+\bar{\omega}_{\gamma+1, \overline{\gamma+1}} e_{\gamma+1}\right\rangle \\
= & \bar{\omega}_{-i,-(\overline{i+1})}\left\langle e_{-(i+1)}, e_{\gamma}\right\rangle .
\end{aligned}
$$


It follows that $\left\langle e_{-(i+1)}, e_{\gamma}\right\rangle=0$ for all $1 \leqslant \gamma \leqslant k$. This completes the induction and the proof.

It follows immediately that $\left\{e_{-l}, \ldots, e_{-1}, e_{1}, \ldots, e_{k}\right\}$ is part of a unitary frame along $x$. In fact it is a unitary framing of the total osculating space of $x$.

Because $\left\{e_{-1}, \ldots, e_{-1}, e_{1}, \ldots, e_{k}\right\}$ spans the total osculating space of $x$, we can choose vector fields $e_{\gamma}, k+1 \leqslant \gamma \leqslant n-l$, so that:

(1) $\left\{e_{-1}, \ldots, e_{-1}, e_{1}, \ldots, e_{k}, e_{k+1}, \ldots, e_{n-1}\right\}$ is a unitary frame along $x$,

(2) $D e_{\gamma}=0$ for $k+1 \leqslant \gamma \leqslant n-l$.

We can assume, then, that $k+l=n$ and that $\left\{e_{-l}, \ldots, e_{-1}, e_{1}, \ldots, e_{k}\right\}$ is a unitary frame along $x$ which satisfies the following relations:

$$
\begin{aligned}
& D e_{-l}=\bar{\omega}_{-l,-i} e_{-l}+\bar{\omega}_{-l,-(\overline{l-1})} e_{-(l-1)} \\
& D e_{-(l-1)}=\bar{\omega}_{-(l-1),-i} e_{-l}+\bar{\omega}_{-(l-1),-(\overline{l-1})} e_{-(l-1)}+\bar{\omega}_{-(l-1),-(\overline{l-2})} e_{-(l-2)}, \\
& \vdots \\
& D e_{-1}=\bar{\omega}_{-1,-\overline{2}} e_{-2}+\bar{\omega}_{-1,-\overline{1}} e_{-1} \\
& D e_{1}=\bar{\omega}_{1 \overline{1}} e_{1}+\bar{\omega}_{1 \overline{2}} e_{2} \\
& D e_{2}=\bar{\omega}_{2 \overline{1}} e_{1}+\bar{\omega}_{2 \overline{2}} e_{2}+\bar{\omega}_{2 \overline{3}} e_{3} \\
& \vdots \\
& D e_{k}=\bar{\omega}_{k, \overline{k-1}} e_{k-1}+\bar{\omega}_{k, \bar{k}} e_{k}
\end{aligned}
$$

where the $\bar{\omega}_{-(\delta-1),-\bar{\delta}}, 2 \leqslant \delta \leqslant l$, are forms (possibly zero) of type $(1,0)$ and the $\bar{\omega}_{\gamma, \overline{\gamma+1}}$, $1 \leqslant \gamma \leqslant k-1$, are forms (possibly zero) of type $(1,0)$.

Definition 3.4. A unitary frame $\left\{e_{-l}, \ldots, e_{-1}, e_{1}, \ldots, e_{k}\right\}$ along a minimal surface in a Kähler manifold of dimension $k+l$ is called a Frenet frame if

(1) $e_{-1}$ and $e_{1}$ are the fields dual to $\omega_{-1}=t \bar{\phi}$ and $\omega_{1}=s \phi$, respectively,

(2) the unitary frame satisfies (3.22).

We have shown that a minimal surface $x: S^{2} \rightarrow X$ which is neither holomorphic nor antiholomorphic admits a Frenet frame if $X$ is a Kähler manifold of constant holomorphic sectional curvature. Recalling the proof of this result we see that we used the fact that the minimal surface is a two-sphere only to conclude that the holomorphic forms $, \ldots \Lambda_{-1}, \Lambda_{0}, \Lambda_{1}, \ldots$ are zero. This motivates the following

DEFINITION 3.5. A minimal surface in a Kähler manifold of constant holomorphic sectional curvature is called superminimal if all the holomorphic forms $\ldots, \Lambda_{-1}, \Lambda_{0}, \Lambda_{1}, \ldots$ are defined and vanish.

The term superminimal was coined by $\mathrm{R}$. Bryant in a somewhat different context [1].

If $X$ is a Kähler manifold of constant holomorphic sectional curvature, then, of course, any minimal two-sphere in $X$ is superminimal. Moreover, we have

THEOREM 3.5. If $X$ is a Kähler manifold of constant holomorphic sectional curvature and $x: M \rightarrow X$ is a superminimal surface, then there is a Frenet frame along $x$.

We remark that although $\Lambda_{0}$ is defined and holomorphic for any minimal surface in $X$ this is not true for the forms $\Lambda_{j}, j \neq 0$. In general $\Lambda_{j}, j>0$, is defined and holomorphic if and only if the $\Lambda_{i}, i=0, \ldots, j-1$, are defined and vanish (similarly for the $\Lambda_{-j}, j>0$ ). Thus on a minimal surface in a Kähler manifold of 
constant holomorphic sectional curvature which is not superminimal there is at least one globally defined holomorphic form (and possibly two different such forms).

It should be remarked that if $X$ is a Kähler manifold nonpositive constant holomorphic sectional curvature, then there are no minimal two-spheres in $X$. It can be shown, however, that locally $X$ admits superminimal surfaces which are neither holomorphic nor antiholomorphic. It is not known if there are any complete superminimal surfaces in $X$ which are neither holomorphic nor antiholomorphic.

4. Minimal surfaces in $\mathbf{C} P^{n}$. We wish to apply the results of $\S 3$ to the case that $X$ is complex projective space, $\mathbf{C} P^{n}$, with the Fubini-Study metric. We begin by giving a description of the geometry of $\mathbf{C} P^{n}$.

For $W, Z \in \mathbf{C}^{n+1}$ the usual Hermitian inner product is defined by

$$
\langle W, Z\rangle=\sum w_{a} \bar{z}_{a}, \quad W=\left(w_{0}, \ldots, w_{n}\right), Z=\left(z_{0}, \ldots, z_{n}\right),
$$

where here and throughout this section we employ the index ranges

$$
0 \leqslant a, b, c \leqslant n, \quad 1 \leqslant i, j, k \leqslant n .
$$

The unitary group $U(n+1)$ is the group of all linear transformations on $\mathbf{C}^{n+1}$ leaving the inner product (4.1) invariant. $\mathbf{C} P^{n}$ is the orbit space of $\mathbf{C}^{n+1}-\{0\}$ under the action of the group $Z \rightarrow \lambda Z$, where $\lambda$ is a complex number $\neq 0$. We thus have the projection map $\pi: \mathbf{C}^{n+1}-\{0\} \rightarrow \mathbf{C} P^{n}$. For a point $p \in \mathbf{C} P^{n}$ a vector $Z \in \pi^{-1}(p)$ is called a homogeneous coordinate vector of $p$. We put $Z_{0}=$ $Z /\langle Z, Z\rangle^{1 / 2}$ so that $\left\langle Z_{0}, Z_{0}\right\rangle=1$. The homogeneous coordinate vector $Z_{0}$ at a point $p \in \mathbf{C} P^{n}$ is defined up to the change

$$
Z_{0} \mapsto \tilde{Z}_{0}=e^{\sqrt{-1} \tau} Z_{0}, \quad \tau \text { real. }
$$

Under this change we have

$$
\begin{gathered}
\left\langle d \tilde{Z}_{0}, \tilde{Z}_{0}\right\rangle=\left\langle d Z_{0}, Z_{0}\right\rangle+\sqrt{-1} d \tau, \\
\left\langle d \tilde{Z}_{0}, d \tilde{Z}_{0}\right\rangle=\left\langle d Z_{0}, d Z_{0}\right\rangle+\sqrt{-1} d \tau\left\{-\left\langle d Z_{0}, Z_{0}\right\rangle+\left\langle Z_{0}, d Z_{0}\right\rangle\right\}+d \tau^{2} .
\end{gathered}
$$

The resulting invariant expression

$$
d s^{2}=\left\langle d Z_{0}, d Z_{0}\right\rangle-\left\langle d Z_{0}, Z_{0}\right\rangle\left\langle Z_{0}, d Z_{0}\right\rangle
$$

is the Fubini-Study metric on $\mathbf{C} P^{n}$.

Now let $Z_{a}$ be a unitary frame in $\mathbf{C}^{n+1}$ so that $\left\langle Z_{a}, Z_{b}\right\rangle=\delta_{a b}$. In the bundle of all unitary frames on $\mathbf{C}^{n+1}$ we have

$$
d Z_{a}=\sum_{b} \psi_{a \bar{b}} Z_{b}
$$

where $\psi_{a \bar{b}}=-\bar{\psi}_{b \bar{a}}=\left\langle d Z_{a}, Z_{b}\right\rangle$ is a one-form. The $\psi_{a \bar{b}}$ are the Maurer-Cartan forms of the group $U(n+1)$ and so satisfy the Maurer-Cartan structure equations

$$
d \psi_{a \bar{b}}=\sum_{c} \psi_{a \bar{c}} \wedge \psi_{\bar{c} \bar{b}}
$$

These are obtained by exterior differentiation of (4.4). By (4.3) and (4.4) the Fubini-Study metric can be written

$$
d s^{2}=\sum_{j} \psi_{0 j} \bar{\psi}_{0 j}
$$


It is of the form (2.1) if we set

$$
\omega_{j}=\psi_{0 j}
$$

If we choose

$$
\omega_{i j}=-\left(\psi_{j i}-\delta_{i j} \psi_{0 \overline{0}}\right),
$$

then these forms satisfy the conditions (2.3) and (2.4). They are therefore the connection forms of the Fubini-Study metric and this metric is Kähler. Its curvature forms are

$$
\Omega_{i j}=-\omega_{i} \wedge \omega_{j}-\delta_{i j} \sum_{k} \omega_{k} \wedge \omega_{\bar{k}}
$$

Thus the Fubini-Study metric has constant holomorphic sectional curvature.

We want to apply these results to an immersed surface $x: M \rightarrow \mathbf{C} P^{n}$. Over a neighborhood $U \subseteq M$ we define a unitary frame $Z_{a}$ along $x$ as $C^{\infty}$ maps

$$
Z_{a}: U \subseteq M \rightarrow \mathbf{C}^{n+1}-\{0\}
$$

such that: (1) $\pi \circ Z_{0}: U \rightarrow \mathbf{C} P^{n}$ is the immersion $x$; and (2) $\left\{Z_{0}, Z_{1}, \ldots, Z_{n}\right\}$ is a unitary frame in $\mathbf{C}^{n+1}$ for each point $p \in U$. All of the above considerations remain valid for a frame field defined in this manner, where we must now regard (4.3)-(4.9) as equations restricted to $M$.

Let $y: M \rightarrow \mathbf{C} P^{n}$ be a holomorphic curve, $U$ a coordinate neighborhood in $M$ and $\zeta$ a complex coordinate on $U$. Using homogeneous coordinates on $\mathbf{C} P^{n}, y$ is given locally by a holomorphic vector valued function $Z(\zeta)=\left(z_{0}(\zeta), \ldots, z_{n}(\zeta)\right)$

$$
Z: U \subseteq M \rightarrow \mathbf{C}^{n+1}-\{0\} .
$$

We assume that $y$ is nondegenerate, i.e. that $y(M)$ does not belong to any hyperplane in $\mathbf{C} P^{n}$. This means that

$$
Z \wedge \frac{\partial Z}{\partial \zeta} \wedge \cdots \wedge \frac{\partial^{n} Z}{\partial \zeta^{n}} \neq 0
$$

except perhaps at isolated points. As $Z$ and its derivatives are all holomorphic functions of $\zeta$, any zeros of (4.11) are removable. This enables us to define a unitary frame along $y$ which is intimately related to the osculating spaces of $y$.

Set $Z_{0}=Z /\langle Z, Z\rangle^{1 / 2}$. Choose maps $Z_{l}: U \subseteq M \rightarrow \mathbf{C}^{n+1}-\{0\}$ such that $Z_{0}(p), Z_{1}(p), \ldots, Z_{l}(p)$ forms a unitary basis for the vector space spanned by $Z(p),(\partial Z / \partial \zeta)(p), \ldots,\left(\partial^{\prime} Z / \partial \zeta^{l}\right)(p)$ (the $l$ th osculating space of $y$ at $\left.p\right)$ for each $l=1, \ldots, n$ and $p \in U .\left\{Z_{0}, \ldots, Z_{n}\right\}$ is a unitary frame along $y$ which satisfies

$$
\begin{aligned}
& d Z_{0}=\psi_{0 \overline{0}} Z_{0}+\psi_{0 \overline{1}} Z_{1}, \\
& d Z_{\sigma}=\psi_{\sigma, \overline{\sigma-1}} Z_{\sigma-1}+\psi_{\sigma, \bar{\sigma}} Z_{\sigma}+\psi_{\sigma, \overline{\sigma+1}} Z_{\sigma+1}, \quad 1 \leqslant \sigma \leqslant n-1, \\
& d Z_{n}=\psi_{n, \overline{n-1}} Z_{n-1}+\psi_{n, \bar{n}} Z_{n},
\end{aligned}
$$

where $\psi_{\rho, \overline{\rho+1}}$ is a form of type $(1,0)$ for $0 \leqslant \rho \leqslant n-1$ and $\psi_{i, \overline{i-1}}$ is a form of type $(0,1)$ for $1 \leqslant i \leqslant n$. 
For later use we record the following

LeMma 4.1. For $0 \leqslant \rho \leqslant n-1$ the $(1,0)$ forms $\psi_{\rho, \overline{\rho+1}}$ have only isolated zeros. In particular, in some neighborhood of $\zeta=0$

$$
\psi_{\rho, \overline{\rho+1}}=\zeta \tilde{h}_{\rho} d \zeta,
$$

where $r$ is an integer $\geqslant 0$ and $\tilde{h}_{\rho}$ is a $C^{\infty}$ function such that $\tilde{h}_{\rho}(0) \neq 0$.

Proof. As $\psi_{\rho, \overline{\rho+1}}$ is a $(1,0)$ form we can write

$$
\psi_{\rho, \overline{\rho+1}}=h_{\rho} d \zeta .
$$

Taking the exterior derivative of (4.14) and using the Maurer-Cartan equations and (4.12) we get

$$
\begin{aligned}
d h_{\rho} \wedge d \zeta & =\psi_{\rho, \bar{\rho}} \wedge \psi_{\rho, \overline{\rho+1}}+\psi_{\rho, \overline{\rho+1}} \wedge \psi_{\rho+1, \overline{\rho+1}} \\
& =\left(\psi_{\rho, \bar{\rho}}-\psi_{\rho+1, \overline{\rho+1}}\right) \wedge h_{\rho} d \zeta
\end{aligned}
$$

So

$$
\left[d h_{\rho}-h_{\rho}\left(\psi_{\rho, \bar{\rho}}-\psi_{\rho+1, \overline{\rho+1}}\right)\right] \wedge d \zeta=0 .
$$

This means that $h_{\rho}$ satisfies a differential equation of the form $(\partial h / \partial \bar{\zeta})=f h_{\rho}$, where $f$ is a complex valued $C^{\infty}$ function on $U$. By Theorem 3.2 either $\psi_{\rho, \bar{\rho}+1}$ satisfies (4.13) or $\psi_{\rho, \overline{\rho+1}} \equiv 0$. The latter implies that the osculating space of $y$ has dimension $\rho$, contradicting the assumption that $y$ is nondegenerate.

A similar statement holds for the $(0,1)$ forms $\psi_{i, i-1}, 1 \leqslant i \leqslant n$.

Each $Z_{i}, 1 \leqslant i \leqslant n$, is determined, by the above construction, up to a transformation $Z_{i} \mapsto \tau Z_{i}$, where $\tau$ is a complex valued function of norm one. If $\pi$ is the projection $\mathbf{C}^{n+1}-\{0\} \rightarrow \mathbf{C} P^{n}$, then it follows that the map

$$
y_{i}=\pi \circ Z_{i}: M \rightarrow \mathbf{C} P^{n}, \quad 1 \leqslant i \leqslant n,
$$

is well defined.

THEOREM 4.1. Suppose $y: M \rightarrow C P^{n}$ is a nondegenerate holomorphic curve. Then the maps

$$
y_{l}: M \rightarrow \mathbf{C} P^{n}, \quad 1 \leqslant l<n,
$$

are branched superminimal immersions which are neither holomorphic nor antiholomorphic. The map $y_{n}: M \rightarrow \mathbf{C} P^{n}$ is an antiholomorphic map.

Proof. Consider the unitary frame $\left\{Z_{0}, \ldots, Z_{l}, \ldots, Z_{n}\right\}$ as a framing along the map $y_{l}$. Set

$$
\begin{aligned}
\omega_{\gamma}=\psi_{l, \overline{l+\gamma}} & \text { for } 1 \leqslant \gamma \leqslant n-l, \\
\omega_{-\delta}=\psi_{l, \overline{l-\delta}} & \text { for } 1 \leqslant \delta \leqslant l .
\end{aligned}
$$

$\left\{\omega_{-\delta}, \omega_{\gamma}\right\}$ is the unitary coframe dual to $\left\{Z_{0}, \ldots, Z_{n}\right\}$. On the surface defined by $y_{l}$ we have

$$
\begin{aligned}
\omega_{\gamma}=0 & \text { for } 2 \leqslant \gamma \leqslant n-l, \\
\omega_{-\delta}=0 & \text { for } 2 \leqslant \delta \leqslant l .
\end{aligned}
$$


The metric on $M$ induced by $y_{l}$ is, then

$$
\begin{aligned}
d s_{y_{l}}^{2} & =\psi_{I, \overline{l+1}} \bar{\psi}_{1, \overline{l+1}}+\psi_{1, \overline{l-1}} \bar{\psi}_{1, \overline{l-1}} \\
& =\omega_{1} \bar{\omega}_{1}+\omega_{-1} \bar{\omega}_{-1} .
\end{aligned}
$$

This is not the same metric as $d s_{y}^{2}=\phi \bar{\phi}$, the metric induced by $y$. However, because $\omega_{1}$ is a $(1,0)$ form and $\omega_{-1}$ is a $(0,1)$ form, the metrics $d s_{y}^{2}$ and $d s_{y_{1}}^{2}$ are conformally equivalent. Thus the complex structures induced on $M$ by $d s_{y}^{2}$ and $d s_{y,}^{2}$ are equivalent. This allows us to write

$$
\omega_{1}=\psi_{1, \overline{1+1}}=s \phi, \quad \omega_{-1}=\psi_{l, \overline{l-1}}=t \bar{\phi}
$$

where $s$ and $t$ are $C^{\infty}$ complex valued functions. (4.17) and (4.18) are the same as equations (2.9) and (2.14). It follows from Lemma 4.1 that the map $y_{l}$ is a branched immersion.

Using the Maurer-Cartan equations to compute the connection forms of the coframe (4.16), we get for $1 \leqslant \gamma \leqslant n-l$ and $1 \leqslant \delta \leqslant l$

$$
\omega_{\gamma,-\bar{\delta}}=-\psi_{l-\delta, \overline{l+\gamma}} \text {. }
$$

From (4.12) it follows that on $M$

$$
\begin{array}{ll}
\omega_{\gamma, \overline{1}}=-\psi_{l-1, \overline{l+\gamma}}=0, & 1 \leqslant \gamma \leqslant n-l, \\
\omega_{-\delta, \overline{1}}=-\psi_{l+1, \overline{l-\delta}}=0, & 1 \leqslant \delta \leqslant l .
\end{array}
$$

Consulting (2.19) and (2.20) we see that this means that $y_{l}: M \rightarrow \mathbf{C} P^{n}$ is minimal. Moreover by the definition of the holomorphic forms $\Lambda_{q},-l+1 \leqslant q \leqslant(n-l)-1$, (4.20) implies that they all vanish. Hence $y_{l}$ is a branched superminimal immersion for $1 \leqslant l<n$.

To see that $y_{n}=\pi \circ Z_{n}$ is an antiholomorphic map, note that since $\psi_{n, \overline{n-1}}$ is a $(0,1)$ form

$$
d Z_{n} \equiv \psi_{n, \bar{n}} Z_{n} \quad \bmod d \bar{\zeta} .
$$

So $d\left(\pi \circ Z_{n}\right) \equiv 0 \bmod d \bar{\zeta}$, i.e. $y_{n}=\pi \circ Z_{n}$ is antiholomorphic.

Theorem 4.1 gives a procedure, involving basically only differentiation, of constructing superminimal surfaces in $\mathbf{C} P^{n}$ from holomorphic curves. Utilizing the Frenet frame of a superminimal surface in $\mathbf{C} P^{n}$ constructed in $\S 3$ we can show that all superminimal surfaces in $\mathbf{C} P^{n}$ can be constructed in this way. In particular, we can show that all minimal two-spheres in $\mathbf{C} P^{n}$ can be constructed using this procedure.

Let $x: M \rightarrow \mathbf{C} P^{n}$ be a nondegenerate superminimal surface. Using homogeneous coordinates on $\mathbf{C} P^{n} x$ is given locally by a vector valued function $Z_{0}: U \subseteq M \rightarrow$ $\mathrm{C}^{n+1}$ of unit length. From the results of $\S 3$ there is a unitary frame $\left\{e_{-l}, \ldots, e_{-1}, e_{1}, \ldots, e_{n-l}\right\}$ along $x$ which satisfies (3.22). We need to translate this framing into a framing using homogeneous coordinates. This is easily accomplished for if $p \in \mathbf{C} P^{n}$ and $V \in \pi^{-1}(p)$, then we can identify $T_{p}\left(\mathbf{C} P^{n}\right)$ with $\left\{W \in \mathbf{C}^{n+1}\right.$ : $\langle W, V\rangle=0\}$. Under this identification we let $e_{-j}, e_{i}$ correspond to $Z_{-j}, Z_{i}$, respectively, for $1 \leqslant j \leqslant l, 1 \leqslant i \leqslant n-l$. Then $\left\{Z_{-l}, \ldots, Z_{-1}, Z_{0}, Z_{1}, \ldots, Z_{n-l}\right\}$ is a unitary frame field along $x$. Moreover, by our convention on the choice of $e_{-1}$ and $e_{1}$ we have

$$
d Z_{0}=\psi_{0,-\overline{1}} Z_{-1}+\psi_{0 \overline{0}} Z_{0}+\psi_{0 \overline{1}} Z_{1},
$$


where

$$
\psi_{0,-\overline{1}}=\omega_{-1}=t \bar{\phi}, \quad \psi_{0 \overline{1}}=\omega_{1}=s \phi .
$$

From (3.22) we know that $\omega_{i j}=0$ if $|i-j|>1$, and from (4.8) we have $\omega_{i j}=$ $-\left(\psi_{j \bar{i}}-\delta_{i j} \psi_{0 \overline{0}}\right)$. Thus

$$
\psi_{i j}=0, \quad|i-j|>1 \text {. }
$$

The unitary frame $\left\{Z_{-l}, \ldots, Z_{-1}, Z_{0}, Z_{1}, \ldots, Z_{n-l}\right\}$ satisfies

$$
\begin{aligned}
& d Z_{-l}=\psi_{-l,-i} Z_{-l}+\psi_{-l,-(\overline{l-1})} Z_{-(l-1)}, \\
& \vdots \\
& d Z_{-1}=\psi_{-1,-\overline{2}} Z_{-2}+\psi_{-1,-\overline{1}} Z_{-1}+\psi_{-1, \overline{0}} Z_{0}, \\
& d Z_{0}=\psi_{0,-\overline{1}} Z_{-1}+\psi_{0, \overline{0}} Z_{0}+\psi_{0 \overline{1}} Z_{1}, \\
& d Z_{1}=\psi_{1, \overline{0}} Z_{0}+\psi_{1 \overline{1}} Z_{1}+\psi_{1 \overline{2}} Z_{2}, \\
& \vdots \\
& d Z_{n-l}=\psi_{n-l, \overline{n-l-1}} Z_{n-l-1}+\psi_{n-l, \overline{n-l}} Z_{n-l}
\end{aligned}
$$

As was the case with the elements of the Frenet frame of a holomorphic curve, each $Z_{i},-l \leqslant i \leqslant n-l$, is determined up to the transformation $Z_{i} \mapsto \tau Z_{i}$, where $\tau$ is a complex valued function of norm one. Thus the maps

$$
x_{i}=\pi \circ Z_{i}: M \rightarrow \mathrm{C} P^{n}
$$

are well defined. By the same argument as in the proof of Theorem 4.1, for $-l+1 \leqslant i \leqslant n-l-1, x_{i}$ is a superminimal surface in $\mathbf{C} P^{n}$. The map $x_{n-l}$ is antiholomorphic and the map $x_{-1}$ is holomorphic. The holomorphic map $x_{-1}$ is called the directrix (or generating) curve of $x$ and is denoted by $\Delta_{x}$.

Comparing (4.24) with (4.12) we see that the framing $\left\{Z_{-l}, \ldots, Z_{0}, \ldots, Z_{n-l}\right\}$ is a Frenet framing of the holomorphic curve $\Delta_{x}$. Moreover, the superminimal surface $x$ occurs as the $l$ th element of this Frenet frame. We have proved the following

THEOREM 4.2. Let $x: M \rightarrow C P^{n}$ be a superminimal surface. Let l be the dimension of the antiholomorphic osculating space of $x$. Then there is a unique holomorphic curve, $\Delta_{x}$, called the directrix curve of $x$ such that $x$ occurs as the lth element of the Frenet frame along $\Delta_{x}$.

Theorem 4.2 shows that all superminimal surfaces in $C P^{n}$ can be constructed using the procedure of Theorem 4.1. Moreover, as minimal two-spheres in $\mathbf{C} P^{n}$ are superminimal, Theorem 4.2 provides a classification of the minimal two-spheres in $\mathbf{C} P^{n}$. That is, a minimal two-sphere in $\mathbf{C} P^{n}$ is determined by its directrix curve (a holomorphic curve in $\mathbf{C} P^{n}$ ) and in the dimension of its antiholomorphic osculating space (an integer between 1 and $n$ ).

5. Further results. In this section we record a few more results of the techniques introduced in $\$ \S 2-4$.

Given a minimal surface $x: M \rightarrow \mathbf{C} P^{n}$ with homogeneous coordinate vector $Z_{0}$, as we have seen, the holomorphic osculating direction $Z_{1}$ and the antiholomorphic osculating direction $Z_{-1}$ give rise to well-defined maps $x_{1}=\pi \circ Z_{1}$ and $x_{-1}=\pi \circ Z_{-1}$ from $M$ to $C P^{n}$. 
THEOREM 5.1. If $x: M \rightarrow \mathbf{C} P^{n}$ is a branched minimal surface, then the maps $x_{1}$ : $M \rightarrow \mathbf{C} P^{n}$ and $x_{-1}: M \rightarrow \mathbf{C} P^{n}$ are also branched minimal surfaces.

The proof follows using the technique of the proof of Theorem 4.1.

This theorem can be extended by considering a minimal surface $x: M \rightarrow \mathbf{C} P^{n}$ for which the forms $\Lambda_{0}=\cdots=\Lambda_{j}=0$ for some $j$ and $\Lambda_{j+1} \neq 0 . x$ is not superminimal; however, we can apply the constructions of $\$ \S 2$ and 3 to find a unitary frame $\left\{Z_{-1}, Z_{0}, Z_{1}, \ldots, Z_{j}, Z_{j+1}, \ldots, Z_{n-1}\right\}$ along $x$ so that

$$
d Z_{\rho}=\psi_{\rho, \overline{\rho-1}} Z_{\rho-1}+\psi_{\rho, \bar{\rho}} Z_{\rho}+\psi_{\rho, \bar{\rho}+1} Z_{\rho+1}, \quad 0 \leqslant \rho \leqslant j,
$$

where $\psi_{\rho, \overline{\rho-1}}$ is a $(0,1)$ form and $\psi_{\rho, \overline{\rho+1}}$ is a $(1,0)$ form. The maps $x_{\rho}=\pi \circ Z_{\rho}$, $-1 \leqslant \rho \leqslant j+1$, are well defined.

THEOREM 5.2. If $x: M \rightarrow \mathbf{C} P^{n}$ is a branched minimal surface for which the forms $\Lambda_{0}=\cdots=\Lambda_{j}=0, \Lambda_{j+1} \neq 0$, then the maps $x_{\rho}=\pi \circ Z_{\rho}: M \rightarrow \mathbf{C} P^{n},-1 \leqslant \rho \leqslant j$ +1 , are also branched minimal surfaces.

Again the proof follows using the technique of the proof of Theorem 4.1. The vanishing of the forms $\Lambda_{0} \cdots \Lambda_{j}$ allows the construction, using basically only differentiation, of $j$ new minimal surfaces. Of course, similar remarks apply to the case $\Lambda_{0}=\Lambda_{-1}=\cdots=\Lambda_{-i}=0$ and $\Lambda_{-(i+1)} \neq 0$.

Consider a minimal surface $x: M \rightarrow \mathbf{C} P^{n}$ for which $\Lambda_{j}=0, j \geqslant 0$. Using the idea $\$ 2$ we can construct a Frenet frame $\left\{Z_{0}, \ldots, Z_{k}\right\}$ for the holomorphic osculating space of $x$. Then the map $x_{k}=\pi \circ Z_{k}: M \rightarrow \mathbf{C} P^{n}$ is an antiholomorphic curve. But then the minimal surface $x$ occurs as an element of the Frenet frame of an antiholomorphic curve. Thus $x$ must be superminimal and the forms $\Lambda_{-j}, j \geqslant 0$, must also vanish. Similarly if $\Lambda_{-j}=0, j \geqslant 0$, then the surface is superminimal and $\Lambda_{j}=0, j \geqslant 0$.

We would like to find results about minimal surfaces in $\mathbf{C} P^{n}$ which relate the geometry of the surfaces to the superminimality conditions. In the case that the surface is the torus, $T^{2}$ (the surface of genus one), we have such a theorem.

The universal covering space of the torus is given by

$$
\begin{gathered}
\text { C } \\
\pi \downarrow \\
T^{2}
\end{gathered}
$$

where $\mathbf{C}$ is the complex plane and $\pi$ is the usual map to $T^{2}$ regarded as $\mathbf{C} / \Gamma, \Gamma$ a lattice in $\mathbf{C}$. We can assume that $\pi$ is a holomorphic mapping. If $\Lambda$ is a holomorphic $(j, 0)$ form on $T^{2}$, then $\pi^{*} \Lambda$ is a holomorphic $(j, 0)$ form on C. Letting $z$ be a global complex coordinate on $\mathbf{C}$ we can write

$$
\pi^{*} \Lambda=g(z)(d z)^{j},
$$

where $g(z)$ is a holomorphic, doubly periodic function on C. It follows that $g$ is constant. 
THEOREM 5.3. If $x: T^{2} \rightarrow \mathrm{C} P^{n}$ is an immersed minimal torus and if for some $p \in T^{2}$ the tangent space $x_{*}\left(T_{p}\left(T^{2}\right)\right)$ is a complex line in $T_{x(p)}\left(\mathbf{C} P^{n}\right)$, then $x$ : $T^{2} \rightarrow \mathbf{C} P^{n}$ is a superminimal surface. Every branched minimal torus in $\mathbf{C P}^{n}$ is superminimal.

Proof. Applying the above remarks to the holomorphic $(3,0)$ form $\Lambda_{0}$ we have $\pi^{*} \Lambda_{0}=g_{0}(z)(d z)^{3}$. Recall that $\Lambda_{0}=\omega_{1} \omega_{\overline{1},-1} \omega_{-\overline{1}}=s \bar{t} \bar{c}(\phi)^{3}$. The condition on the tangent space of the immersed minimal torus (resp. the condition that $p \in T^{2}$ is a branch point) says with respect to our normalization (2.14)

$$
\omega_{1}=s \phi, \quad \omega_{-1}=t \bar{\phi},
$$

that either $s(p)=0$ or $t(p)=0$ (resp., both $s(p)=0$ and $t(p)=0$ ). It follows that $g_{0}\left(\pi^{-1}(p)\right)=0$. But $g_{0}$ is a constant. Hence $\pi^{*} \Lambda_{0}=0$; and so $\Lambda_{0}=0$.

The vanishing of $\Lambda_{0}$ implies that $\Lambda_{1}$ (and $\Lambda_{-1}$ ) is defined and holomorphic. Moreover, if we set $\omega_{1 \overline{2}}=u \bar{\phi}$ and $\omega_{2,-\overline{1}}=v \bar{\phi}$, where $u$ and $v$ are complex valued functions, then

$$
\Lambda_{1}=\omega_{1} \omega_{\overline{1} 2} \omega_{\overline{2},-1} \omega_{-\overline{1}}=\operatorname{su} \bar{v} \bar{t}(\phi)^{4}
$$

Thus, as above, $\Lambda_{1}=0$.

Continuing, this argument shows that all the $\Lambda_{j}$ 's, $j=0, \pm 1, \pm 2, \ldots$, vanish.

We conclude this section by indicating how Calabi's classification of minimal two-spheres in the Euclidean sphere $S^{N}[2,3]$ can be derived from our result. Let $w$ : $S^{2} \rightarrow S^{N}$ be a minimal immersion, where $S^{N}$ is the sphere of radius 1 in Euclidean $(N+1)$-space. We assume that $w$ is nondegenerate in the sense that the image of $w$ does not lie in an equator of $S^{N}$. Denote by pr: $S^{N} \rightarrow \mathbf{R} P^{N}$ the projection of $S^{N}$ to real projective $N$-space and denote by $\iota: \mathbf{R} P^{N} \rightarrow \mathbf{C} P^{N}$ the inclusion. The map $\iota \circ \mathrm{pr}$ is totally geodesic, so the map $x=\imath \circ \mathrm{pr} \circ w: S^{2} \rightarrow \mathrm{C} P^{N}$ is a minimal immersion

$$
x: S^{2} \stackrel{w}{\rightarrow} S^{N} \stackrel{\mathrm{pr}}{\rightarrow} \mathbf{R} P^{N} \stackrel{\iota}{\rightarrow} \mathbf{C} P^{N} .
$$

Furthermore, $x$ is real (i.e., $x=\bar{x}$ ) and nondegenerate (i.e. $x\left(S^{2}\right)$ does not lie in a hyperplane).

Applying the results of $\$ 4$ to the minimal surface $x$ we get a Frenet frame $\left\{Z_{-l}, \ldots, Z_{-1}, Z_{0}, Z_{1}, \ldots, Z_{k}\right\}$ along $x$, where $\left\{Z_{0}, \ldots, Z_{k}\right\}$ is a Frenet frame for the holomorphic osculating space of $x$ and $\left\{Z_{0}, \ldots, Z_{-l}\right\}$ is a Frenet frame for the antiholomorphic osculating space of $x . \pi \circ Z_{-l}$ is the directrix curve, $\Delta_{x}$, of $x$. As $x$ is real, we have $k=l$ and

$$
Z_{-i}=\bar{Z}_{i}, \quad 1 \leqslant i \leqslant l=k .
$$

In particular $\pi \circ Z_{-k}=\bar{\Delta}_{x}$.

Proposition 5.1. If $w: S^{2} \rightarrow S^{N}$ is a nondegenerate minimal immersion, then $N$ is even.

Proof. Using the above notation we have by the nondegeneracy of $x, k+l=N$. But as $x$ is real, $k=l$.

We can suppose then that $N=2 l$. 
Because the holomorphic curve $\Delta_{x}$ is associated with a minimal map $S^{2} \rightarrow S^{2 l}$ it has some special properties. To explain these we denote by $($, ) the scalar product on $\mathbf{C}^{2 l+1}$ which is complex linear in both arguments. That is, if $Y, Z \in \mathbf{C}^{2 l+1}$, $Y=\left(y_{0}, y_{1}, \ldots, y_{2 l}\right), Z=\left(z_{0}, z_{1}, \ldots, z_{2 l}\right)$ then

$$
(Y, Z)=\sum_{A=0}^{2 l} y_{A} z_{A} \text {. }
$$

For $1 \leqslant i, j \leqslant l$ we have by $(5.2)$,

$$
0=\left\langle Z_{i}, Z_{-j}\right\rangle=\left\langle Z_{i}, \bar{Z}_{j}\right\rangle=\left(Z_{i}, Z_{j}\right)
$$

The osculating space of order $j$ of $\Delta_{x}$ for $1 \leqslant j \leqslant l$ is

$$
S_{j}=Z_{-l} \wedge Z_{-l+1} \wedge \cdots \wedge Z_{-l+j}
$$

It follows from (5.3) that the $(l-1)$ osculating space of $\Delta_{x}$ lies completely on the nondegenerate hyperquadric $Q_{2 l-1}$ in $\mathbf{C} P^{2 l} \cdot Q_{2 l-1}$ is defined by the equation $(Z, Z)=0$, where $Z$ is a homogeneous coordinate vector in $\mathbf{C}^{2 l+1}$. Moreover, it is not difficult to show that any nondegenerate holomorphic curve $\Delta: S^{2} \rightarrow \mathrm{C} P^{2 l}$, whose $(l-1)$ osculating space satisfies this property, is the directrix curve associated to some minimal map $S^{2} \rightarrow S^{2 l}[7,10]$.

\section{REFERENCES}

1. R. L. Bryant, Every compact Riemann surface may be immersed conformally and minimally into $S^{4}$, J. Differential Geom. 17 (1982), 455-473.

2. E. Calabi, Minimal immersions of surfaces in Euclidean spheres, J. Differential Geom. 1 (1967), $111-125$.

3. __ Quelques applications de l'analyse complexe aux surfaces d'aire minima, Topics in Complex Manifolds, University of Montreal, 1967, pp. 59-81.

4. S. S. Chern, On minimal spheres in the four sphere, Studies and Essays presented to Y. W. Chen, Taiwan, 1970, pp. 137-150; also, Selected Papers, Springer-Verlag, New York, 1978, pp. 421-434.

5. Analysis, Princeton Univ. Press, Princeton, N. J., 1970, pp. 27-40.

6. S. S. Chern, M. J. Cowen and A. L. Vitter, Frenet frames along holomorphic curves, Proc. Conf. on Value Distribution Theory, Tulane University, 1974, pp. 191-203.

7. S. S. Chern and J. G. Wolfson, Minimal surfaces by moving frames, Amer. J. Math. 105 (1983), 59-83.

8. A. M. Din and W. J. Zakrzewski, General classical solutions in the $\mathbf{C} P^{n-1}$ model, Nuclear Phys. B 174 (1980), 397-406.

9. J. Eells and J. C. Wood, Harmonic maps from surfaces to complex projective spaces, Advances in Math. 49 (1983), 217-263.

10. J. G. Wolfson, Minimal surfaces in complex manifolds, Ph.D. Thesis, University of California, Berkeley, 1982.

Department of Mathematics, Rice University, Houston, Texas 77251

Current address: Department of Mathematics, Tulane University, New Orleans, Louisiana 70118 